\title{
Autonomous generator based on Ni-Mn-Ga microactuator as a frequency selective element
}

\author{
M. M. Krupa ${ }^{1}$, Y. B. Skirta ${ }^{1}$, J.M. Barandiaran ${ }^{2}$, M. Ohtsuka ${ }^{3}$, V.A. Chernenko ${ }^{2,4}$ \\ ${ }^{1}$ Institute of Magnetism, NASU, Vernadsky Blvd., 36-b, Kyiv 03142, Ukraine \\ ${ }^{2}$ Universidad del País Vasco, UPV/EHU, P.O. Box 644, 48080 Bilbao, Spain \\ ${ }^{3}$ IMRAM, Tohoku University, Sendai 980-8577, Japan \\ ${ }^{4}$ Ikerbasque, Basque Foundation for Science, 48011 Bilbao, Spain
}

\begin{abstract}
In this work, we suggest the temperature-induced resistivity change at the martensitic transformation in the Ni-Mn-Ga ferromagnetic shape memory alloy as a driving mechanism enabling periodic signal generation. We demonstrated its practical importance by a design of the prototype of a low-frequency autonomous generator. A prominent feature of this new generator is a control of its frequency by the external magnetic field.
\end{abstract}

\section{Introduction}

Ni-Mn-Ga alloys are multiferroics as they exhibit two types of the strongly correlated long-range order: ferroelasticity and ferromagnetism. The martensitic structure and martensitic transformation (MT) in these materials are the core ingredients of their extraordinary properties and multifunctionality as they can be controlled by a magnetic field. Particularly, the magnetoelastic, ordinary shape memory, and magnetoresistance actuation properties near MT have been already explored and implemented into the prototype devices [1-3].

The resistivity anomaly accompanying MT in Ni-MnGa alloys has $N$-type shape and its amplitude, like transformation temperature $T_{\mathrm{m}}$, drastically depends on the composition [4]. When MT occurs in the ferromagnetic austenite, a magnetic field shifts $T_{\mathrm{m}}$ upwards [5], this shift being about ten times larger in a value for MT merged with Curie temperature, $T_{\mathrm{C}}[6]$. In addition, NiMn-Ga thin films deposited on $\mathrm{Al}_{2} \mathrm{O}_{3}$ or glass substrates demonstrate a thickness dependence of both $T_{\mathrm{m}}$ and corresponding resistivity anomaly $[7,8]$.

In this work, we found a good cyclic reversibility of MT and accompanying low-hysteretic temperature dependence of resistivity exhibited by Ni-Mn-Ga film deposited on alumina substrate. The autonomous generator was constructed where the Ni-Mn-Ga thin film microactuator was served as a frequency convertor element.

\section{$2 \mathrm{Ni}-\mathrm{Mn}-\mathrm{Ga} / \mathrm{Al}_{2} \mathrm{O}_{3}$ thin film actuator}

The Ni-Mn-Ga thin film with a thickness of $5 \mu \mathrm{m}$ deposited at room temperature on the alumina ceramic plate of $150 \mu \mathrm{m}$ thickness was fabricated previously by RF magnetron sputtering using target $\mathrm{Ni}_{49.5} \mathrm{Mn}_{28} \mathrm{Ga}_{22.5}$ (see Refs.[7,8] for details). The samples of $5 \times 5 \mathrm{~mm}^{2}$ were cut from the alumina plate with the film and annealed at $1273 \mathrm{~K}$ in vacuum of $2 \times 10^{-4} \mathrm{~Pa}$ for $3.6 \mathrm{ks}$ with a subsequent slow cooling in a furnace, for homogenization and ordering. This standard procedure alongside the same processing parameters as used in Refs. [7,8] facilitates the martensitic transformation in the film below $T_{\mathrm{C}}$ with a formation of the tetragonal $10 \mathrm{M}$ martensitic phase stable at the room temperature.

The transformation behavior of Ni-Mn-Ga/ $\mathrm{Al}_{2} \mathrm{O}_{3}$ microactuator was studied by recording its resistance versus temperature curves. The value of $T_{\mathrm{C}}$ and temperature characteristics of MT appear to be similar to the previous results [7] while $N$-type resistance anomaly itself is steeper and has lower hysteresis, see the data in the next section. The similar resistivity behaviour with negative temperature coefficient exhibit the well-known thermistors which are widely commercially used in engineering, particularly, as a frequency convertors in the low-frequency generators. This fact served us as a motivation to develop an autonomous generator based on $\mathrm{Ni}-\mathrm{Mn}-\mathrm{Ga}$ microactuator which frequency could be remotely controlled by a magnetic field.

\section{Design and performance of generator}

Block diagram of autonomous generator is presented in figure 1. The bridge circuit, consisting of Ni-Mn-Ga microactuator element (Rs) and constant resistors R1, R2 
and R3 is a frequency convertor which is the principal part of generator. The heater $\left(R_{h}\right)$ consisting of planar resistors which are in a good thermal contact with the film substrate, serves for thermal actuation of the film. The copper-constantan thermocouple (1) and semiconducting thermometer, LM-335 (2) are needed for the temperature control of the film and room temperature, respectively. The ICP-CON digital-analog conversion modules, i-7017 and i-7018 (3), as well as a relay modulus i-7060 (4) are used for voltage measurements and heater control, respectively. All modules are connected with a personal computer through an interface RS-485 (5).

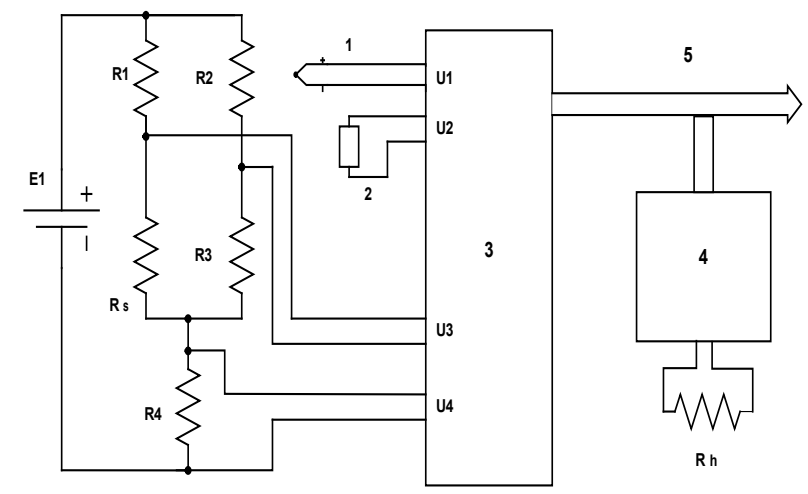

Fig. 1. Block diagram of autonomous generator. E1 is DC current source; Ni-Mn-Ga microactuator element $\left(\mathrm{R}_{\mathrm{s}}\right)$ and constant resistors R1, R2 and R3 form bridge circuit generating a signal when $\mathrm{R}_{\mathrm{s}}$ is periodically heated by a heater $\mathrm{R}_{\mathrm{h}}$; $\mathrm{R} 4$ is reference resistor; 1 and 2 are thermocouple and semiconducting thermometer for registration of the temperatures of sample and environment, respectively; 3 and 4 are the digital-analog conversion modules and a relay modulus, respectively; 5 is an interface between modules and PC.

The values of the sample resistance $R_{\mathrm{S}}$ are calculated by using voltage at the bridge diagonal and at the reference resistor:

$R_{s}=I_{1}\left(R_{2}+R_{3}\right) /\left(I-I_{1}\right)-R_{1}$

where

$I=U_{4} / R_{4}$ and $I_{1}=\left(U_{3}+I R_{1}\right) /\left(R_{2}+R_{1}\right)$

In these expressions, the symbols $I$ and $U$ hold for the current and voltage, respectively.

We set the working temperature range of Ni-Mn-Ga microactuator, $R_{\mathrm{s}}$, corresponding to its martensitic and austenitic finish temperatures: $M_{\mathrm{f}}=313.2 \mathrm{~K}$ (the heater turns on) and $A_{\mathrm{f}}=323.3 \mathrm{~K}$ (the heater turns off), into the control program. This enables a pre-heating of the sample and protects against overheating in the case of a fault. First, sample is heated to the working temperature. After that, the generator searches for the inflection point on the $R=\mathrm{f}(T)$ curve that corresponds to the end of austenitic transformation. In this point, the heater turns off. Then, the sample is naturally cooled and transforms martensitically until the point that corresponds to the finish of martensitic transformation. In the latter point, the heater turns on. After that, the generator cycle is repeated. Figures 2 and 3 show example of one generator heating-cooling cycle in the terms of $R=\mathrm{f}$ (time,t) and $R=\mathrm{f}(T)$ for the heater power of $1.125 \mathrm{~W}$. The active segment (heater on) is marked in red, passive segment (heater off) is drawn in black. The heater is switched on and off at the corresponding inflection point on the curve. There is small delay between switching the heater and the start of cooling or heating. This delay is caused by a finite value of the thermal conductivity.

We found that cycle period and waveform depend on films properties, heater power and the value of room temperature. Other stimuli such as mechanical stress pressure and/or magnetic field should obviously influence too.

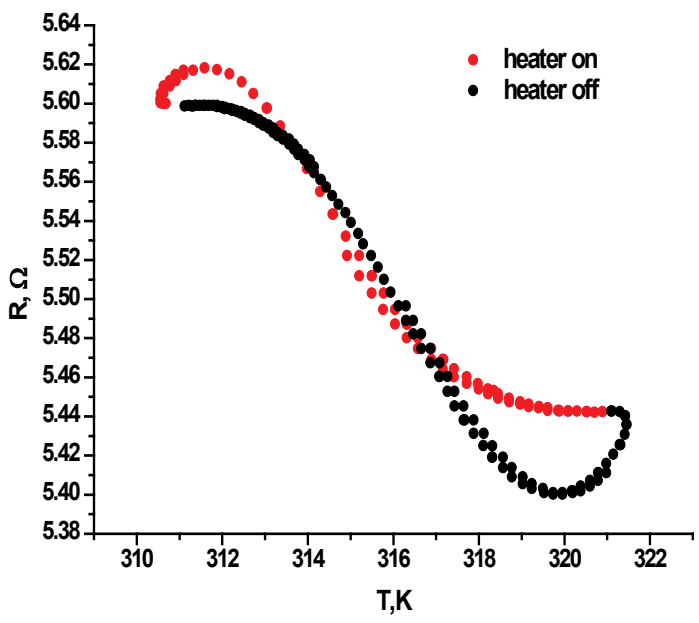

Fig. 2. One cooling-heating cycle of resistance, $R(T)$, of Ni-MnGa microactuator undergoing a martensitic and an austenitic transformation, respectively. The heater power is $1.125 \mathrm{~W}$

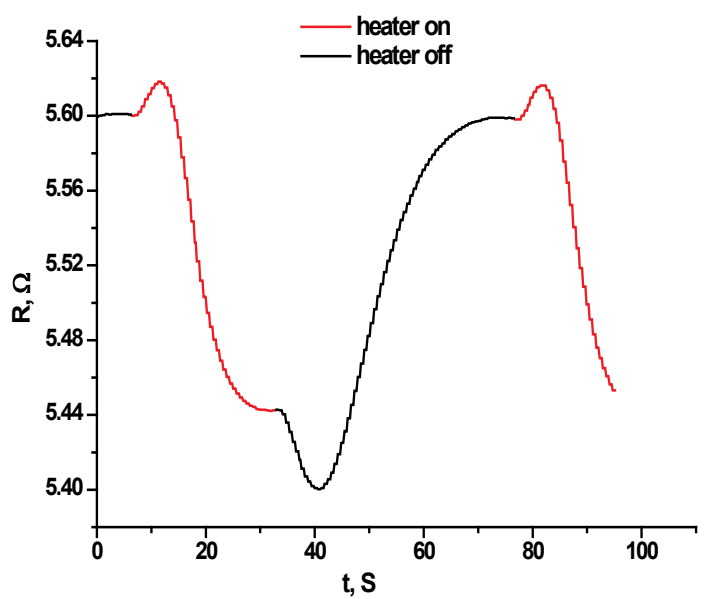

Fi

g.3. The same cycle as shown in figure 2 in terms of heatingcooling time period. The heater power is $1.125 \mathrm{~W}$.

In the current work, we have used three different values of the heater powers. We found that the device goes into a stable regime, repeats amplitude, period and waveform of the signal, if the room temperature and heater power are constant and there are no external fields. 
Table 1. Performance of the generator at three heating powers

\begin{tabular}{|c|c|c|}
\hline $\begin{array}{c}\text { Heater power, } \\
W\end{array}$ & Signal period, $\mathrm{s}$ & $\begin{array}{c}\text { Signal frequency, } \\
\mathrm{Hz}\end{array}$ \\
\hline 1.125 & $71.2 \pm 1.7$ & 0.014 \\
\hline 0.91 & $91.2 \pm 1.8$ & 0.011 \\
\hline 0.72 & $129.8 \pm 2.6$ & 0.008 \\
\hline
\end{tabular}

A stable regime of the oscillations, in the case of heating power of $1.125 \mathrm{~W}$, is demonstrated in figure 4 . The corresponding period of a signal obtained is $71.2 \pm 1.7 \mathrm{~s}$, as also indicated in Table 1 . Table 1 compiles the performance of generator at the mentioned three different heating powers.

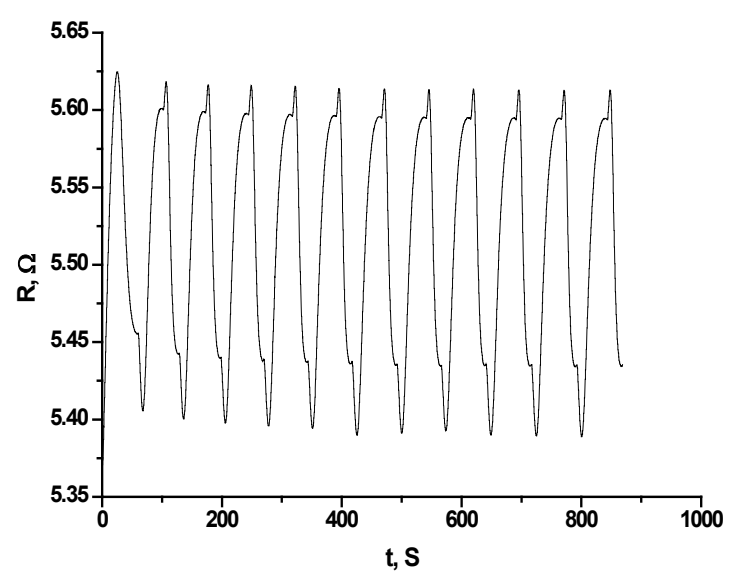

Fig.4. The time dependence of the Ni-Mn-Ga microactuator resistance at heating power of $1.125 \mathrm{~W}$.

All measurements were performed at room temperature $T=296 \mathrm{~K}$. The averaging was performed for 25 generator cycles. The first cycle was excluded because its waveform was significantly distorted. The calculated averaged values of heating-cooling rates are 8.5, 6.6 and $4.7 \mathrm{~K} / \mathrm{min}$ corresponding to three descending values of powers in Table 1, respectively.

We made preliminary measurements of the influence of magnetic field on the generator performance. A magnetic field of 17.5 Tesla was applied perpendicularly to the Ni-Mn-Ga microactuator. The shifts of $\Delta M_{\mathrm{f}}$ $=(0.48 \pm 0.16) \mathrm{K}$ and $\Delta A_{\mathrm{f}}=(1.43 \pm 0.17) \mathrm{K}$ have been observed. These shifts result in a decrease of signal frequency by about $9.5 \%$. So, the well-known effects of positive shifts of transformation temperatures under magnetic field and/or stress in Ni-Mn-Ga $[5,6,8]$ will inevitably result in a decrease of the frequency.

On the other hand, the signal frequency can be effectively increased by the enhancement of the heat exchange rate between actuator/heater assembly and ambient atmosphere. This can be done by both decreasing size of the active elements and/or by varying surrounding medium.

\section{Conclusions}

It is shown for the first time that the very low-frequency autonomous generator $(0.01 \mathrm{~Hz})$ can be developed based on the shape memory materials exhibiting low-hysteretic martensitic transformation with $N$-shaped segment on the $R=\mathrm{f}(T)$ dependence. The developed oscillator can be used as a scientific instrument for accurate transformation temperature measurements, study of the transformation dynamics and precise determination of the influence of the external fields such as magnetic field and/or mechanical stress, on the transformation temperatures.

We have shown that the oscillator characteristics can be remotely operated by a magnetic field in the case of ferromagnetic shape memory alloys which extends new dimension in exploration of their multifunctionality. The magnetic field control implies that suggested lowfrequency generation principle can be of advantage in comparison to one based on thermistor properties [9]. This should be further confirmed by detailed study of the influence of magnetic field on the oscillator frequency which is under the way.

Practical importance of the suggested oscillator in the engineering can be enforced by simplifying its block diagram which is feasible if $\mathrm{Ni}-\mathrm{Mn}-\mathrm{Ga}$ or other magnetic shape memory materials with a larger resistivity anomaly at the martensitic transformation are used.

A finite elements approach is planned to be used for the optimization of the generator performance.

\section{Acknowledgements}

The financial support from the Basque Government (Projects: Actimat, ETORTEK IE10-272 and Research Groups funding, IT 304-07) and the Spanish Ministry of Science and Innovation (Project MAT2011-28217-C0202 ) is acknowledged.

\section{References}

1. D. Auernhammer, M. Kohl, B. Krevet, M. Ohtsuka, Smart Mater. Struct. 18,104016 (2009).

2. I. Suorsa, J. Tellinen, E. Pagounis, I. Aaltio, and K. Ullakko, Proc. 8th Int. Conf. on Actuators, Bremen, Germany, 10-12 June 2002, p. 158.

3. I. Suorsa, J. Tellinen, K. Ullakko, E. Pagounis, J.Appl.Phys. 95,8054 (2004).

4. V. A. Chernenko, Scr. Mater., 40, 523(1999).

5. V. A. Chernenko, V. A.Lvov, T. Kanomata, T. Kakeshita, K. Koyama, S. Besseghini, Mat. Trans. 47, 635 (2006).

6. V. V. Khovailo,V. Novosad, T. Takagi, D. A. Filippov, R. Z. Levitin, A. N. Vasil'ev, Phys. Rev.B70, 174413 (2004).

7. V. A. Chernenko, M. Ohtsuka, M. Kohl, V. V. Khovailo, T. Takagi, Smart Mater. Struct. 14, S245 (2005).

8. V. A. Chernenko, M. Kohl, V. A. L'vov, V. M. Kniazkyi, M. Ohtsuka, O.Kraft, Mater. Trans. 47, 619 (2006).

9. E. D. Macklen, Thermistors (Electromechanical Publication Ltd., 1979). 